\title{
Early life microbial colonization of the gut and intestinal development differ between genetically divergent broiler lines
}

\author{
Dirkjan Schokker ${ }^{1 *}$, Gosse Veninga ${ }^{2}$, Stephanie A. Vastenhouw ${ }^{3}$, Alex Bossers ${ }^{3}$, Freddy M. de Bree ${ }^{3}$, \\ Lucia M. T. E. Kaal-Lansbergen', Johanna M. J. Rebel ${ }^{3}$ and Mari A. Smits ${ }^{1}$
}

\begin{abstract}
Background: Host genetic makeup plays a role in early gut microbial colonization and immune programming. Interactions between gut microbiota and host cells of the mucosal layer are of paramount importance for a proper development of host defence mechanisms. For different livestock species, it has already been shown that particular genotypes have increased susceptibilities towards disease causing pathogens.

The objective of this study was to investigate the impact of genotypic variation on both early microbial colonization of the gut and functional development of intestinal tissue. From two genetically diverse chicken lines intestinal content samples were taken for microbiota analyses and intestinal tissue samples were extracted for gene expression analyses, both at three subsequent time-points (days 0, 4, and 16).

Results: The microbiota composition was significantly different between lines on each time point. In contrast, no significant differences were observed regarding changes in the microbiota diversity between the two lines throughout this study. We also observed trends in the microbiota data at genus level when comparing lines $X$ and $Y$. We observed that approximately 2000 genes showed different temporal gene expression patterns when comparing line $X$ to line $Y$. Immunological related differences seem to be only present at day 0, because at day 4 and 16 similar gene expression is observed for these two lines. However, for genes involved in cell cycle related processes the data show higher expression over the whole course of time in line $Y$ in comparison to line $X$.

Conclusions: These data suggest the genetic background influences colonization of gut microbiota after hatch in combination with the functional development of intestinal mucosal tissue, including the programming of the immune system. The results indicate that genetically different chicken lines have different coping mechanisms in early life to cope with the outside world.
\end{abstract}

Keywords: Gut, Chicken, Immune, Microbiota, Gene expression

\section{Background}

During the last decades, growth performance traits were the main driver for the genetic selection programs applied for broilers. This already underscores the importance of genetic factors contributing to this important economic trait for poultry production. More recently the knowledge that intestinal microbiota also play a key role in growth-related traits such as feed digestibility, feed

\footnotetext{
* Correspondence: dirkjan.schokker@wur.nl

${ }^{1}$ Wageningen Livestock Research, Wageningen, The Netherlands
}

Full list of author information is available at the end of the article uptake, protein fermentation, but also in health related traits such as immune competence and immune tolerance. For human and mice data have been described that suggest a role for early-life colonizing microbiota in host immune programming [1-5], yet for chicken such data are lacking. Furthermore the impact of genotypic variation on early life microbial colonization in relation to the functional development of the gut is largely unknown. In fact, the gut microbiota is regarded a metabolic powerhouse that provides the functionally limited host with an extensive array of enzymes and substrates required for growth. It has been shown that gut microbiota can

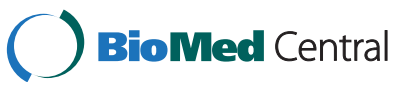

(c) 2015 Schokker et al.; licensee BioMed Central. This is an Open Access article distributed under the terms of the Creative Commons Attribution License (http://creativecommons.org/licenses/by/4.0), which permits unrestricted use, distribution, and reproduction in any medium, provided the original work is properly credited. The Creative Commons Public Domain Dedication waiver (http://creativecommons.org/publicdomain/zero/1.0/) applies to the data made available in this article, unless otherwise stated. 
influence energy retention and can predispose to obesity $[6,7]$. Additionally, they play a role in the renewal of gut epithelial cells and its barrier function $[8,9]$, the breakdown of toxins [10], the exclusion of pathogens [11], and the programming and development of the immune system [12]. The aim of this research was to investigate the impact of genetic background of these broiler lines on the microbial colonization and the development of the small intestine in early life.

In chickens, microbiota colonization occurs immediately after hatch, both microbiota from the egg shell and environment form the first inoculum of the chickens [13]. The characteristics of this first inoculum is of utmost importance because it impacts the further colonization of microbiota and simultaneously the functional development of the intestinal tissue in terms of barrier function and in terms of immune programming [13, 14]. It has been shown that around day 14 of age a presumably more stable microbiota is not yet established [15], however, immunological development in the small intestine has already occurred [16-18]. Around day 14, intestinal segments have different functional properties and harbour distinctive microbiota compositions [19].

In chickens, not much is known about the effect of host genetic background on microbial colonisation and microbiota composition. It has, however, been shown that high variation occurs in microbial composition between individual chickens and flocks of genetically closely related animals [20]. For mice it has been demonstrated that the host genetic background has impact on the microbiota composition in the gut [21]. However, it still difficult to disentangle the relationship between host genetic factors and the microbiota composition directly [22]. Nevertheless, first attempts have been made in mice to search for quantitative trait loci (QTL) associated to the presence and/or abundance of specific bacterial species or taxa and evidence is provided that host genetic control occurs in shaping the microbiota composition [23].

In poultry, not much is known about the effect of the genetic background on intestinal immune development. It is known that at hatch the innate and adaptive immune systems are immature and functional maturation occurs mainly the first 2 weeks of life in broilers $[16,18]$. The innate immunity is the first line of defence, which includes the barrier function (epithelial layers), complement and coagulation cascade, phagocytes (e.g. macrophages), natural killer cells, and dendritic cells. Besides the role of the epithelial layer being the first line of defence against invading pathogens, these cells also play an important role in maintaining intestinal homeostasis by integration of microbial signals (reviewed in [24]). The adaptive immune system is also described as the acquired immune system, in which memory cells are generated after the initial challenge with an antigen. These memory cells facilitate a more efficient and faster response upon subsequent challenges with the same antigen.

It has been shown that restricting microbial exposure of chicken during early life has impact on mucin production [25]. Mucins are key components of epithelial layers, serving functions from lubrication to cell signalling to forming barriers to chemicals and pathogens. These observations already imply that bacterial colonization and critical aspects of gut (immune) homeostasis and/or barrier functions are intertwined with each other. Furthermore, studies on the effects of different pig rearing environments showed a negative effect of more 'hygienic' environments during early life on immune development [26]. Similarly, early life use of antibiotics in piglets has been shown to alter microbiota colonization as well as immune development [27]. It is proposed that a proper immune development requires colonization with "natural" microbiota present in the future environment [28].

The objective of this study was to investigate the impact of genetic background on both early microbial colonization of the gut in combination with functional development of intestinal tissue. To accomplish this, community-scale analysis of gut lumen microbiota and genome-wide transcriptome profiling of intestinal tissue was used. In this study we compared two genetically divergent chicken lines that differ from each other in health (immune) related phenotypes, in particular in bacterial infections and the related pathology and severity of such induced diseases by differing in immune response against Salmonella enteritidis, vancomycin-resistant Enterococcus, and Campylobacter jejuni [29-35]. The justification for using these selected lines was that they had known differences in susceptibility towards bacterial infections and thus may differ as well in the basic level of immune competence. To identify similar and dissimilar functional processes in time between the two chicken lines, the temporal gene expression profiles were analysed in more detail.

\section{Results}

\section{Performance data}

Body weight (Fig. 1) and Feed Conversion Ratio (FCR; Table 1) were measured in time, in order to generate a representative view of the whole life of a broiler. No statistical significant differences were observed per time-period between lines $\mathrm{X}$ and $\mathrm{Y}$ for both body weight and FCR. Body weight increased from approximately 100 grams on day 4 to approximately $2.3 \mathrm{~kg}$ on day 36 . The FCR was slightly higher in line $\mathrm{X}$ compared to line $\mathrm{Y}$ at each time-point.

\section{Microbiota analyses}

To investigate the differences between the two broiler lines regarding their microbiota, both redundancy (Fig. 2) and diversity analyses (Fig. 3) were performed. The redundancy analysis showed that the primary discriminant 


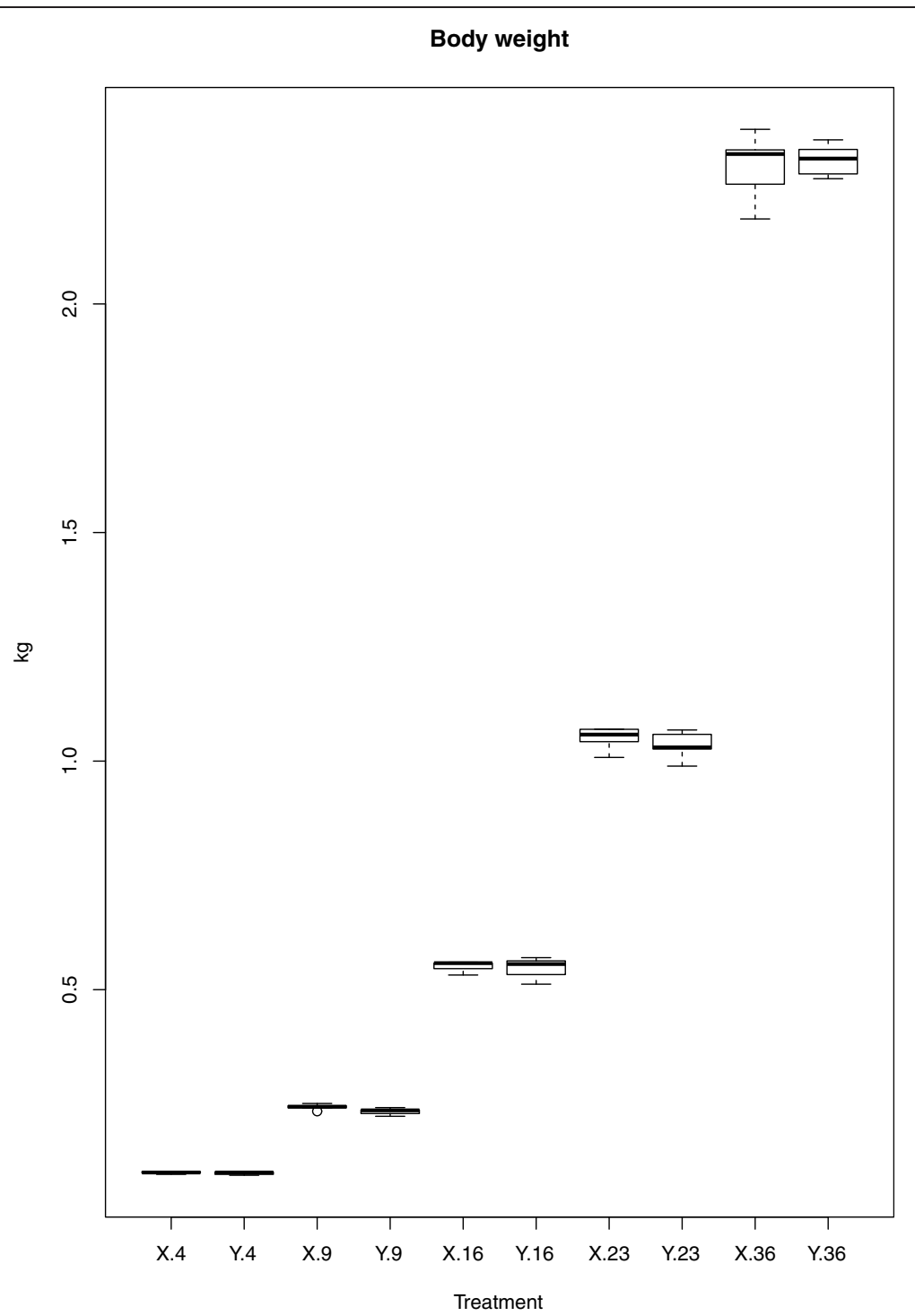

Fig. 1 Boxplot of body weight in time for each chicken line. On the $X$-axis the lines $X$ and $Y$ and the days are depicted and the $y$-axis shows the body weight in kilograms. The letters $X$ and $Y$ stand for the chicken line and the numbers for the age in days

on the microbiota composition between the two genetically different broiler lines was the factor time, and secondary was the influence of genotype. An ANOVA permutation test for RDA under a reduced model, where terms were added sequentially (first to last) showed that

Table 1 Difference in feed conversion ratios in time between chicken line $X$ and $Y$

\begin{tabular}{lcc}
\hline Time-period & Line $Y-X$ & Ratio in \% \\
\hline $0-9$ & -0.004 & 99.46 \\
$9-16$ & -0.037 & 96.93 \\
$16-23$ & -0.001 & 99.91 \\
$23-36$ & -0.001 & 99.91 \\
\hline
\end{tabular}

Age, Line, and Age:Line were all significant, p-values 0.01, 0.03 , and 0.01 , respectively.

In the diversity analysis, which was calculated by the Shannon-index, we observed an increase in time of the microbiota diversity for each chicken line. However, no statistically significant differences were observed between the lines, in other words similar levels of diversity were observed between the two lines ( $\mathrm{X}$ and $\mathrm{Y}$ ).

To investigate to what extent the relative abundance of specific bacterial species were different between the chicken lines, averages of microbial families and/or species were calculated for each time-point separately (Table 2). At day 0 lines $\mathrm{X}$ and $\mathrm{Y}$ have different abundance levels of the 2 to 3 most dominant bacterial genera, Enterococci, Escherichia and Lactobacilli. Day 4 after 


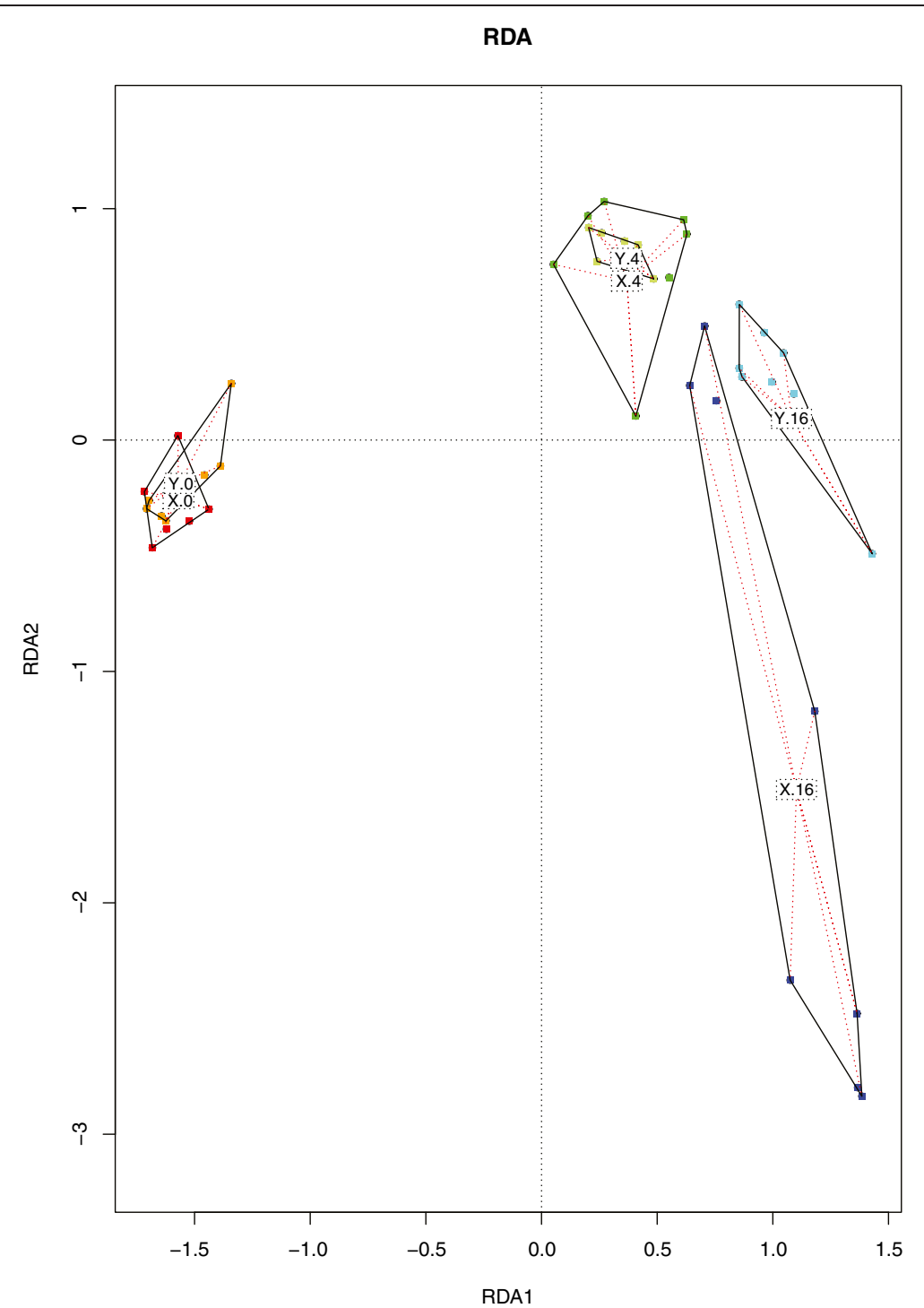

Fig. 2 Redundancy analysis of jejunal microbiota composition (family level) on day 0, 4, and 16. Each symbol represents a pool of 10 chickens and represents their average microbiota composition. The data are represented as follows, for line X day 0 (red), day 4 (green), day 16 (blue) and for line Y day 0 (orange), day 4 (light green), day 16 (cyan)

hatch shows that the abundance of bacterial genera are more closely together, and the highest abundance is of Lactobacilli, $88 \%$ average relative contribution (ARC) in line $\mathrm{X}$ and $84.5 \%$ ARC in line Y. Also at day 16 similar ARC abundance at the bacterial genera level were observed between lines $\mathrm{X}$ and $\mathrm{Y}$, however line $\mathrm{Y}$ had two specific genera, Enterococci and Escherichia, which were markedly higher compared to line X. Yet line X displayed overall higher ARC in various bacterial genera.

\section{Transcriptome analyses}

First an explorative principal component analysis (PCA) was performed, in which the different time-points and broiler lines could be separated (Fig. 4). In this analysis only the first two components were taken into consideration, because the variance explained was already $98.05 \%$. This PCA shows a convergence in overall gene expression in time between the two lines. At day 0 , lines $\mathrm{X}$ and $\mathrm{Y}$ are clearly separated on both principal component 1 (PC1) and $\mathrm{PC} 2$, whereas on day 4 and 16 separation of the two lines was mainly on PC2.

To get more insight into differentially expressed genes between the two lines on each time-point, statistical testing by LIMMA was performed in which up- and downregulation was also taken into account (Table 3; upper part). Subsequent to the LIMMA analysis, functional annotation clustering (DAVID) was performed to highlight particular processes that differ between lines $\mathrm{X}$ and $\mathrm{Y}$ 


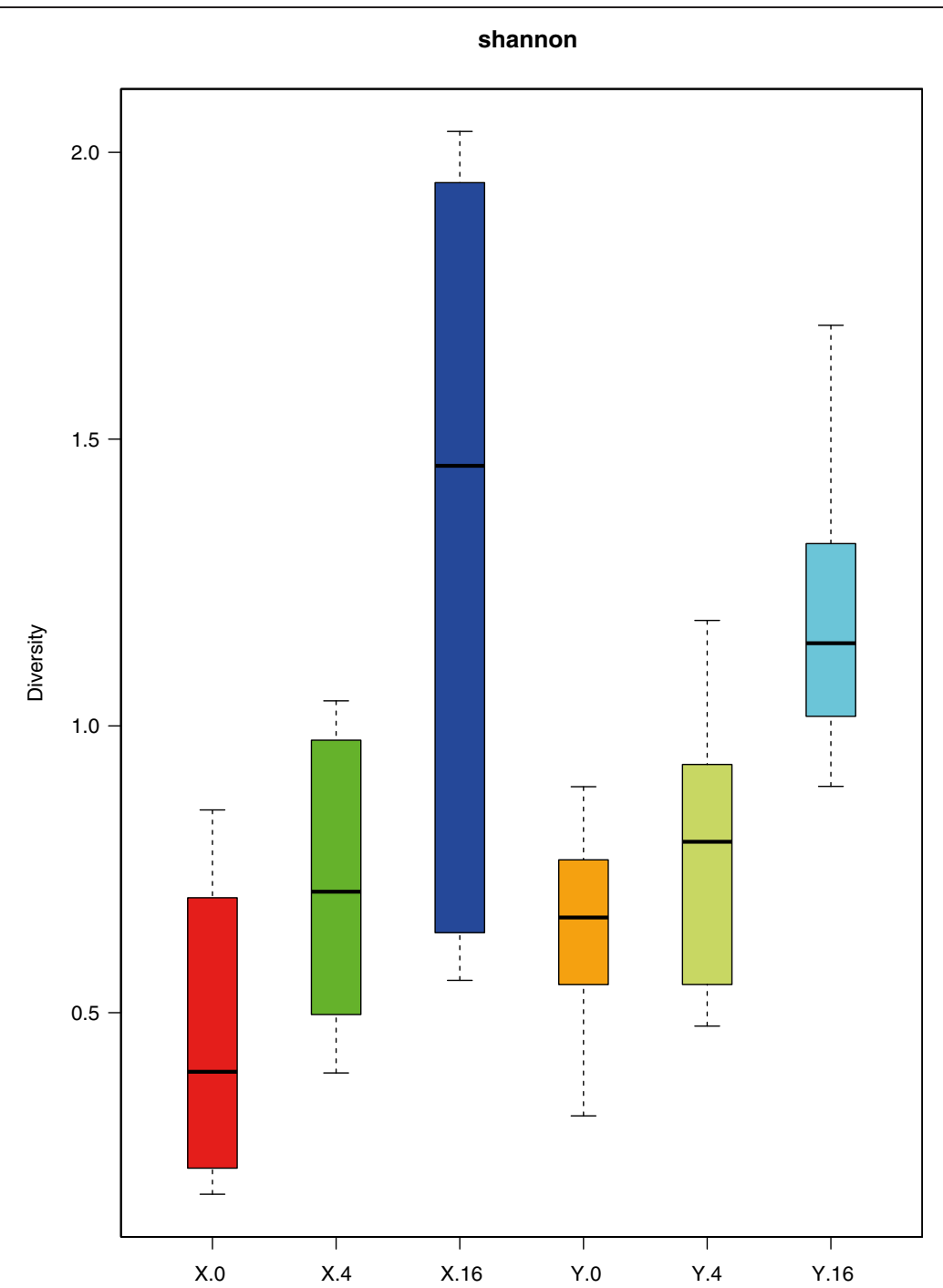

Fig. 3 Microbiota diversity analysis of jejunal microbiota composition on day 0,4 , and 16. Box plot of the microbiota diversity measured by the Shannon index. The data are represented as follows, for line X day 0 (red), day 4 (green), day 16 (blue) and for line Y day 0 (orange), day 4 (light green), day 16 (cyan)

(Table 3; lower part). All enrichment scores (ES) above 1.3 of a particular functional annotation cluster were taken into account for day 0, 4, and 16 separately. Each 'enriched' cluster was summarized manually into twothree representative keywords describing the generic term (Table 4).

To investigate the differences in temporal gene expression patterns between the lines, a regression based approach was performed (R-package maSigPro). This resulted in 3671 probes, corresponding to 1922 genes, that had different temporal expression patterns over time. The next step was to identify (dis)similar temporal expression patterns between the lines, therefore 'soft' clustering of the genes was performed (R-package MFuzz). Nine clusters were generated, each displaying a particular expression pattern in time for one or both lines (Table 5).
Visualization of these cluster is shown in Fig. 5, were red lines depict line $\mathrm{X}$ and green lines depict line $\mathrm{Y}$. Furthermore, to investigate the function of the collection of genes in a certain cluster, functional annotation clustering was performed and the results are depicted in Fig. 5.

\section{Discussion}

In this study two genetically different broiler lines were compared, which were known to differ in their immunological responses towards bacterial infections and the pathology and severity of the induced diseases [29-35]. However, these broiler lines did not differ in any growth characteristics. Thus these broiler lines may differ in the basic level of immune competence. Currently it is not known on what level the two lines deviate from each other and what the underlying mechanisms are for their 
Table 2 Most abundant microbiota ${ }^{a}$ between lines $X$ and $Y$

\begin{tabular}{|c|c|c|c|c|c|c|c|c|c|}
\hline$\overline{P^{b}}$ & Class & Family & Genus & $X .0$ & Y.0 & $X .4$ & Y.4 & $X .16$ & Y.16 \\
\hline \multirow[t]{9}{*}{$\mathrm{F}$} & \multirow[t]{5}{*}{ Bacilli } & \multirow[t]{2}{*}{ Enterococcaceae } & Enterococcus & 83.5 & 71 & 0.16 & 1.33 & 0.09 & 0.72 \\
\hline & & & Other & 1.12 & 1.28 & 7.74 & 8.6 & 3.2 & 6.12 \\
\hline & & Lactobacillaceae & Lactobacillus & 0.12 & 0.02 & 88 & 84.5 & 68.8 & 72.9 \\
\hline & & Streptococcaceae & Streptococcus & 0 & 0 & 1.6 & 2.45 & 14 & 15.1 \\
\hline & & Bacillaceae & Other & 0 & 0 & 0 & 0 & 1.48 & 0.01 \\
\hline & \multirow[t]{3}{*}{ Clostridia } & Other & Other & 0 & 0 & 0.14 & 0.09 & 0.926 & 0.04 \\
\hline & & Lachnospiraceae & Other & 0 & 0 & 0.12 & 0.02 & 1.44 & 0.11 \\
\hline & & Ruminococcaceae & Faecalibacterium & 0 & 0 & 0 & 0 & 0.741 & 0.01 \\
\hline & Erysipelotrichi & Erysipelotrichaceae & Coprobacillus & 0 & 0 & 0.02 & 0 & 1.5 & 0.01 \\
\hline $\operatorname{Pr}$ & Gammaproteobacteria & Enterobacteriaceae & Escherichia & 12.6 & 26.8 & 1.47 & 2.69 & 1.98 & 0.84 \\
\hline T & Mollicutes & & & 0 & 0 & 0 & 0 & 1.26 & 0.03 \\
\hline
\end{tabular}

above $0.1 \%$ average relative contribution in at least one condition

${ }^{2} \mathrm{P}$, Phylum; F, Firmicutes; Pr, Proteobacteria; T, Tenericutes

${ }^{3}$ In bold make up $>95 \%$ of the average relative contribution in a certain condition

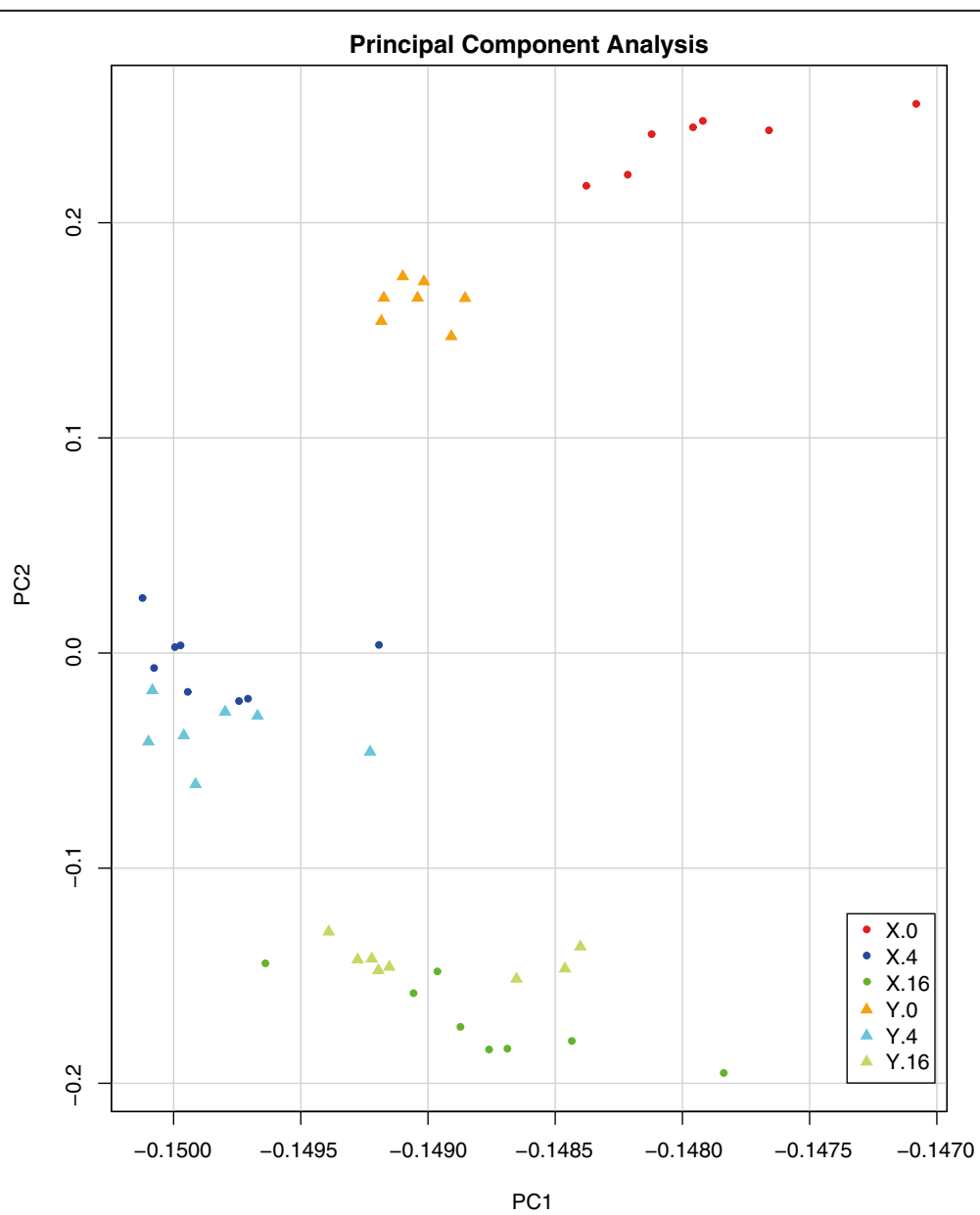

Fig. 4 Principal Components Analysis of jejunal transcriptomics data of chicken lines $X$ and $Y$. Each symbol represents whole-genome gene expression data (approximately $44 \mathrm{k}$ probes) of a pool of 10 chickens. The data are represented as follows, for line X (circles) day 0 (red), day 4 (blue), day 16 (green) and for line Y (triangles) day 0 (orange), day 4 (cyan), day 16 (light green) 
Table 3 Differential expression between groups and corresponding functional annotation clustering

\begin{tabular}{|c|c|c|c|c|c|c|}
\hline \multirow[t]{2}{*}{ LIMMA } & \multicolumn{2}{|l|}{$Y .0-X .0$} & \multicolumn{2}{|l|}{ Y.4-X.4 } & \multicolumn{2}{|l|}{ Y.16-X.16 } \\
\hline & Down & Up & Down & Up & Down & Up \\
\hline Number of probes ${ }^{a}$ & 283 & 741 & 70 & 449 & 171 & 232 \\
\hline Number of genes ${ }^{a}$ & 109 & 418 & 33 & 256 & 80 & 89 \\
\hline \multirow[t]{2}{*}{ DAVID } & Y.O-X.0 & & Y.4-X.4 & & Y.16-X.16 & \\
\hline & Down & Up & Down & Up & Down & Up \\
\hline DAVID identifiers ${ }^{b}$ & 70 & 334 & 21 & 194 & 64 & 53 \\
\hline Number of clusters & 18 & 104 & 5 & 69 & 19 & 11 \\
\hline$E S>1.3$ & 3 & 6 & 0 & 7 & 3 & 0 \\
\hline
\end{tabular}

adjusted $p$-value $<0.05$ and absolute fold change $>1.3$

${ }^{b}$ from the human (hsa) database

Table 4 Generic biological terms ${ }^{a}$ from Functional Annotation Clustering (ES > 1.3) between line $X$ and $Y$

\begin{tabular}{|c|c|}
\hline \multicolumn{2}{|l|}{ Day 0 - low in $Y$ vs. high in $X$} \\
\hline Generic Term ${ }^{\mathrm{a}}$ & ES \\
\hline serine proteinase/complement/resp. wounding & 1.57 \\
\hline Protease/peptidase activity & 1.47 \\
\hline LIM/Zinc finger & 1.47 \\
\hline \multicolumn{2}{|l|}{ Day 0 - high in $Y$ vs. low in $X$} \\
\hline Generic Term & ES \\
\hline mitochondrial & 2.87 \\
\hline organelle lumen (nitracellular/nucleus) & 2.84 \\
\hline intracellular protein transport & 2.46 \\
\hline protein localization & 2.3 \\
\hline mitochondrion outer membrane & 1.52 \\
\hline nuclear pore & 1.35 \\
\hline \multicolumn{2}{|l|}{ Day 4 - high in $Y$ vs. low in $X$} \\
\hline Generic Term & ES \\
\hline translation & 2.28 \\
\hline lumen/nucleus & 2.15 \\
\hline mitochondrion & 1.79 \\
\hline mitochondrion outer membrane & 1.72 \\
\hline lipase activity & 1.32 \\
\hline regulation of translation & 1.3 \\
\hline cytoskeleton/microtubule & 1.3 \\
\hline \multicolumn{2}{|l|}{ Day 16 - low in $Y$ vs. high in $X$} \\
\hline Generic Term & ES \\
\hline ion transport & 2.46 \\
\hline channel activity & 1.58 \\
\hline anion transport & 1.34 \\
\hline
\end{tabular}

${ }^{a}$ Manually curated the database hits per cluster into as few as possible representative key words difference in immunological properties. It is known that programming of the innate as well as the adaptive immune system occurs mainly at young age and the microbial species in the gastro-intestinal tract play a major role in shaping the immune system and the development of the intestinal barrier functions [36, 37]. Compared to human and mice, in chicken not much is known about the impact of genotypic variation on early life microbial colonization in relation to the functional development of the gut. The objective of this study was to investigate the impact of the genetic variation between the two broiler lines on both microbial colonization of the gut and functional development of intestinal tissue. The microbial communities of the two lines ( $\mathrm{X}$ and $\mathrm{Y}$ ) differ in composition, but have similar levels of diversity. Also the intestinal gene expression patterns showed marked differences between the two broiler lines, especially at days 0 and 4 .

\section{Microbiota differences in two genetically different broiler lines}

In this experiment both broiler lines were hatched at the same hatchery and before starting this experiment the chambers were disinfected with the same protocols. Furthermore, both broiler lines were hatched and reared at the same time (in separate pens) and chickens were exposed to the same environment and nutrition This might explain the small differences observed in the diversity of microbiota, this in agreement with the concept that environmental microbiota are the first colonizers [20,38]. The composition of microbiota is dependent on the circumstances in the gut and therefore it seems logical to assume that host genetic factors have a bigger impact on microbiota composition than on microbiota diversity. Therefore we hypothesize that the observed difference in microbiota composition over time is due to the fact that these broilers are genetically different. The importance of host genetic factors has already been observed in mice and humans $[22,39,40]$. Since the differences between the lines $\mathrm{X}$ and $\mathrm{Y}$ in microbiota composition are already demonstrable at the early life stage of the birds, this may have impact on immune programming and the activity of the immune system at later life stages. This hypothesis is in agreement with previous observation with these lines that their immunological responses upon challenge are quite different [29-35]. These studies showed that line $Y$ was more immunological responsive and resistant against both Gram-negative (Salmonella) and Gram-positive bacteria (vancomycin-resistant Enterococcus gallinarum), as well as parasitic protozoan (Eimeria tenella). In recent work, they have proven that chickens with a higher phenotype of key pro-inflammatory mediators (IL-6, CXCLi2 (IL8L2), and MIP family CC chemokine CCLi2) were naturally more resistant against Salmonella [41]. The latter shows that it is possible to select for natural resistance against 
Table 5 Results of soft clustering of genes displaying a different temporal expression pattern when comparing line $X$ and $Y$

\begin{tabular}{|c|c|c|c|c|c|c|c|c|c|c|}
\hline Cluster & 1 & 2 & 3 & 4 & 5 & 6 & 7 & 8 & 9 & Total \\
\hline Number of probes & 190 & 900 & 486 & 382 & 524 & 314 & 323 & 240 & 312 & 3,671 \\
\hline Number of genes & 99 & 371 & 295 & 274 & 199 & 156 & 189 & 123 & 216 & 1,922 \\
\hline
\end{tabular}

pathogens, here we want to understand the dynamics and kinetics in early development in more detail in order to improve the resistance against pathogens in chicken.

In order to understand the dynamics and kinetics of the colonization, we zoomed in on the major differences in microbiota composition between the lines included Enterococci, Lactobacilli, and Escherichia species. Enterococci belong to the lactic acid bacteria, members of the gastro-intestinal tract of hosts, due to the fact that they can colonize a range of hosts including Caenorhabditis elegans, insects, reptiles, birds, and mammals [42, 43]. Enterococci faecalis and E. faecium are the two most abundant commensals in humans. However, these species are also potential pathogens and contain intrinsic (and acquired) mechanisms for antibiotic resistance and virulence factors (reviewed in [44]) and for this reason

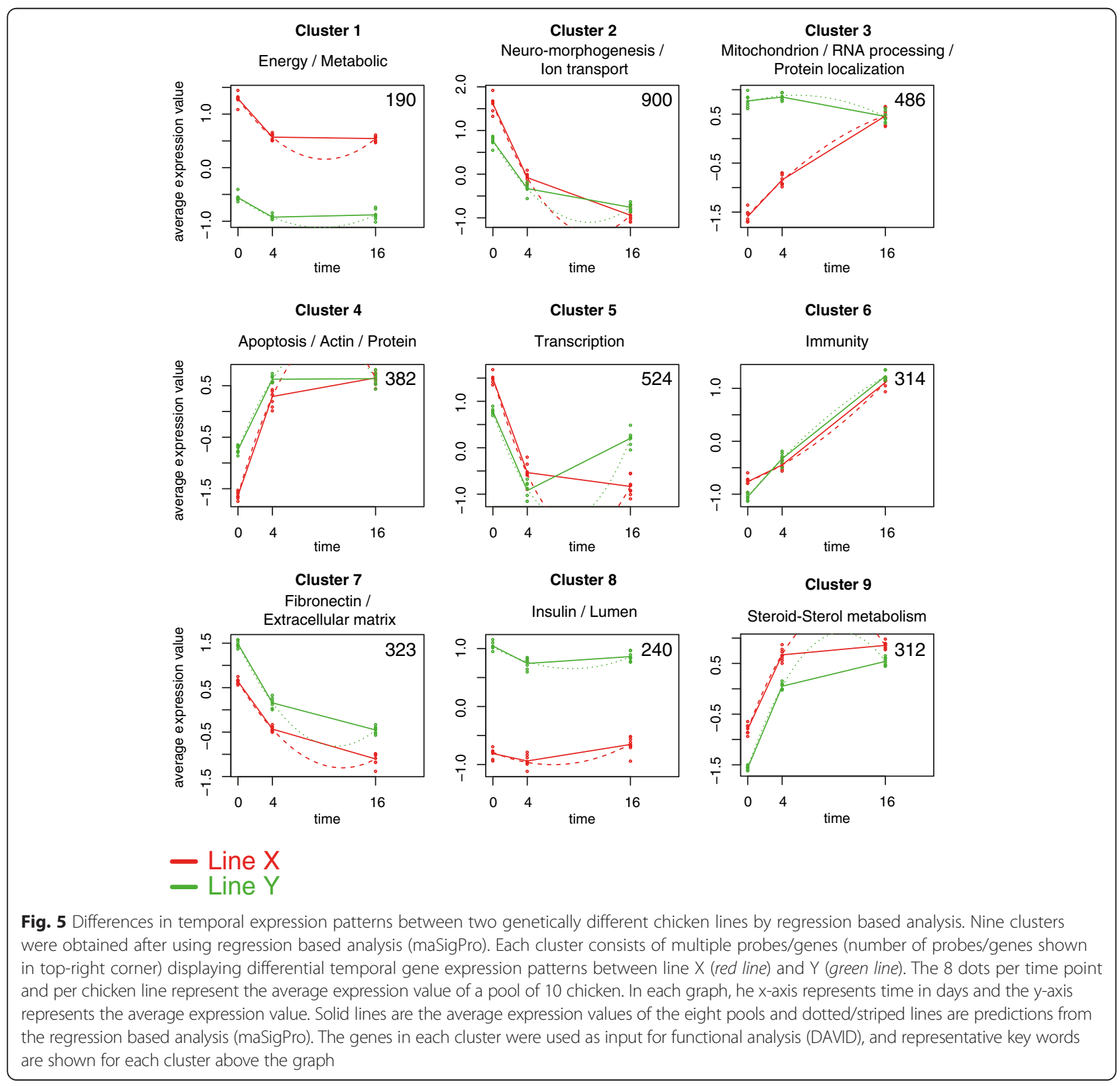


they are now labelled as important clinical pathogens. Their specific contribution to the programming and shaping of the immune system is thus far not known.

Escherichia, facultative anaerobe bacteria, are another genus that is dominant in early life of these broilers. In adult pigs they have shown that high numbers Escherichia, mostly Escherichia coli, appear in the gastro-intestinal tract, nonetheless the health status was not affected in these pigs [45]. Although the role of commensal E. coli in the gastrointestinal tract is not yet fully understood, we need to acknowledge that $E$. coli is dominant in early life and could fulfil an important role in the development of the gut ecosystem.

Lactobacilli are characterized as beneficial for health, mainly because they produce lactic acid which inhibits the growth of (putative) pathogens. Different Lactobacillus strains are already identified that exclude other (pathogenic) bacteria by competitive exclusion or enhancing immunity. For example, Lactobacillus salivarius prevents Salmonella enteritidis colonization CTC2197 [46]. It has also been shown that a cocktail of bacteria, including Lactobacillus acidophilus, Lactobacillus casei, Bifido bacterium bifidium, and Enterococcus faecium, enhances mucosal immunity against Eimeria acervulina [47]. Another example is the Lactobacillus johnsonii FI9785 bacteria which excludes bacterial pathogens [48]. However, Lactobacilli can become pathobionts, but this is mostly in immuno-compromised or elderly people [49]. Different strains of Lactobacilli are also used as probiotics to improve the health status of humans [50-53] and chickens [54-56]. This shows, that a higher abundance of (particular) Lactobacilli strains in the gut may affect health status beneficially [54-56], either by reducing the pathogen load and/or strengthening the immune defence against infections. In this research, the time factor is predominantly present throughout the data, i.e. the colonization of the Lactobacilli from 0-0.1\% ARC at day 0 to $84-88 \%$ ARC at day 4. This high relative contribution of Lactobacilli over time may also be important for the immune development in the gut. Only small differences between line $\mathrm{X}$ and $\mathrm{Y}$ are observed, where line $\mathrm{X}$ has slightly higher abundance at day 0 , which could be associated to the high activity of immune genes.

\section{Different temporal gene expression patterns between broiler lines}

The expression of many genes and processes in the intestinal tissue differed between the two broiler lines. The most dominant processes were related to cell cycle and proliferation. Another cluster was associated to immunity, whereas other clusters could not be linked to immunity or barrier function, such as metabolic and transcriptional processes.
The link of genes being involved in cell cycle and proliferation is shown in several clusters $(3,4$, and 7$)$. More specifically these clusters were associated to apoptosis, extracellular matrix, and mitochondrion. These results show that cell proliferation/apoptosis is a main difference between these lines, especially in early age, these processes may be directly linked to the barrier function of the gut. It has been estimated that every 3-5 days the gut is renewed [57], tight regulation of cell death (apoptosis) and proliferation of epithelial cells is paramount to ensure structural integrity in the gut [58]. In chicken multiple interventions, including fasting, diet, thermal conditioning, and stress, already have been described that affect epithelial structure, growth, and function [59-62].

Moreover, the intestinal barrier has an important role in maintaining homeostasis and when dysfunction of the intestinal barrier occurs this is associated to several gastrointestinal diseases, including inflammatory bowel disease, food intolerance, and celiac disease [63-66]. Compared to line $\mathrm{X}$, line $\mathrm{Y}$ showed higher average expression of cell cycle and proliferation related processes which may contribute to the immune phenotype observed, higher resistance against bacterial infections. This data suggests that a heightened status of the barrier function in early life could lead to less mortality. This could also be reflected in the lower activity of immune genes at day 0 in line $Y$, indicating that the immune programming might be delayed.

We were surprised to see limited effects on immune related processes. Only one cluster differing between line $\mathrm{X}$ and $\mathrm{Y}$ was associated with immunity, one could expect more because these lines differ in resistance against bacterial infection and showed deviating gene expression after an immune challenge [29-35]. This immunity cluster only differed at day 0 , where line $\mathrm{X}$ showed higher expression compared to line $\mathrm{Y}$ and from day 4 to 16 a similar expression pattern was observed. This immunological expression pattern in time was already observed in earlier work of our group and others [16-18]. This suggests that overall immune development in these broiler lines were comparable except at early stage. Thus, in order for chickens to survive the turbulent start of life which is accompanied with the microbial colonization and gut differentiation, chickens may either invest in the immune programming or the barrier function at an early stage.

\section{Conclusions}

- Both colonization of gut microbiota and functional processes are influenced by the genetic background

- Microbiota composition diverges over time

- Intestinal functional processes and corresponding gene expression are mainly affected in early life between two genetically different chicken lines 
- Temporal differences between these two lines suggest different coping mechanisms in early life

\section{Methods \\ Experimental design}

1-day-old chicks (Lines X and Y) were housed in a floor pen system in which the chicks had ad libitum access to feed and water. At days 0, 4 and 16, 80 birds of each line $\mathrm{X}$ and line $\mathrm{Y}$ were sacrificed for tissue sampling, in total 240 birds per line. Subsequently, samples from these 80 birds were pooled in 8 pools of ten birds. Samples were pooled because our main interest was in the generic processes at the population level of these genetically divergent broiler lines. We acknowledge that we lose the ability to interpret the data on an individual level. This pooling strategy, 8 pools of 10 chicken, enabled us to identify smaller differences between the two genetically different broiler lines compared to analysing 8 individual chickens. The absolute difference in the average of individual samples appointed significant was 1.3 , whereas in the pooling strategy this was reduced to 0.4 .

The Feed Conversion Ratio (FCR) was calculated by dividing the feed intake for the time-period of interest by the average daily gain for the time-period of interest. The following time-periods were analysed 0-9, 9-16, 1623, and 23-36, and each time the FCR for line X was subtracted from line $\mathrm{Y}$ to investigate the difference between the broiler lines.

\section{Ethics statement}

This animal experiment was approved by the institutional animal experiment committee "Dier Experimenten Commissie (DEC) Lelystad" (accession number 2013035.b), in accordance with the Dutch regulations on animal experiments.

\section{Microbiota analysis DNA extraction}

Jejunal content was snap frozen in liquid nitrogen and stored at $-80{ }^{\circ} \mathrm{C}$. For the microbial DNA extraction the following protocol was used. Jejunal content was mixed 1:1 with PBS and vortexed, spun for $5 \mathrm{~min}(300 \mathrm{~g})$ at $4{ }^{\circ} \mathrm{C}$. The supernatant was transferred to a new tube and spun for $10 \mathrm{~min}(9000 \mathrm{~g})$ at $4{ }^{\circ} \mathrm{C}$, thereafter supernatant was removed. Subsequently the QIAamp DNA Stool Mini Kit protocol was used as described by the manufacturer (https://www.qiagen.com/nl/products/catalog /sample-technologies/dnasample-technologies/genomic-dna /qiaamp-dna-stool-mini-kit/). The samples were eluted in $100 \mu \mathrm{l}$ of the (provided) elute buffer and thereafter an optical density measurement was performed to check the DNA quality on Nanodrop (Agilent Technologies).

\section{V3 $P C R$}

Oligo V3_F, with sequence; CCTACGGGAGGCAGCAG and oligo V3_R with sequence; ATTACCGCGGCTGCT GG.

The following PCR program was ran: Step $198{ }^{\circ} \mathrm{C}$ for 2 min. Step $298{ }^{\circ} \mathrm{C}$ for 10 s, step $355^{\circ} \mathrm{C}$ for 30s, and step $472{ }^{\circ} \mathrm{C}$ for 10 s, step $572{ }^{\circ} \mathrm{C}$ for $7 \mathrm{~min}$. Where step 2-4 were repeated for 15 times. All PCR products were subjected to quality control by running $5 \mu$ of PCR

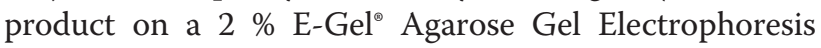
System (Life Technologies).

\section{QIIME}

Samples were sequenced by targeted-amplicon $16 \mathrm{~S}$ sequencing on the MiSeq (Additional file 1: Table S1 shows the reads per sample and Additional file 1: Table S2 shows the summary per broiler line at each time-point) and analysed for taxonomy profile per sample with clustering by profile by using QIIME [67]. Standard assembly based on amplicon, with primer removal was performed. For Quality filtration the following settings were used: 1 ) > Q20 and 2) amplicons $>100$ bases. For the data analysis pseudoreads were clustered into operational taxonomic units (OTUs) per sample at $97 \%$ similarity and OTUrepresentative sequences were aligned against the aligned Greengenes core set (13_8 release) [68, 69]. Singletons were removed, as well as chimeras, the latter with Chimeraslayer [70].

\section{Statistical analysis}

The biodiversity was calculated by the vegan package (http://cran.r-project.org/web/packages/vegan/) within the $R$ environment, by employing the Shannon diversity index. The richness and evenness data (together with the diversity) are depicted in Additional file 1: Tables S3, S4 and S5. The Redundancy analysis (RDA) was also performed by using the vegan package, the following model was ran on the family level microbiota data: $y=$ Time + Treatment + Time* Treatment + error. Furthermore, statistical significance testing for over- and underrepresentation of the bacterial groups was made at the family level by performing the Wilcoxon signed-rank test, and $\mathrm{p}$-values were converted to false discovery rate (FDR) values to correct for multiple testing.

\section{Transcriptomic analysis RNA extraction tissue}

Total RNA was extracted from 50 to $100 \mathrm{mg}$ jejunum tissue. All samples were homogenised using the TisuPrep Homogenizer Omni TP TH220P) in TRizol reagent (Life Technologies) as recommended by the manufacturer with minor modifications. The homogenised tissue samples were dissolved in $5 \mathrm{ml}$ of TRizol reagent. After centrifugation the supernatant was transferred to a fresh tube. 
Subsequently a phase separation with chloroform was performed as described by the manufacturer Life Technologies. The RNA was precipitated and dissolved and quantified by absorbance measurements at $260 \mathrm{~nm}$. Quality Control was performed using the Agilent Bioanalyser.

\section{Labelling, hybridization, scanning and feature extraction} Labelling of RNA was done as recommended by Agilent Technologies using the One-Color Microarray-Based Gene Expression Analysis Low input Quick Amp Labelling. The input was $10 \mathrm{ng}$ of total RNA and $600 \mathrm{ng}$ of labelled cRNA was used for hybridization on the eight pack array (Agilent 049577 chicken array). Hybridization was performed as described in the One-Color MicroarrayBased Gene Expression Analysis Low input Quick Amp Labelling protocol from Agilent in the hybridization oven (G2545A hybridization Oven Agilent Technologies). The hybridization temperature was $65{ }^{\circ} \mathrm{C}$ with rotation speed $10 \mathrm{rpm}$ for $17 \mathrm{~h}$. After $17 \mathrm{~h}$ the arrays were washed as described in the One-Color Microarray-Based Gene Expression Analysis Low input Quick Amp Labelling protocol from Agilent. The arrays were scanned using the DNA microarray scanner with Surescan high resolution Technology from Agilent Technologies. Agilent Scan Control with resolution of $5 \mu \mathrm{m}, 16$ bits and PMT of $100 \%$. Feature extraction was performed using protocol 10.7.3.1 (v10.7) for 1 colour gene expression.

\section{Data analysis}

The gene expression data (GEO accession number GSE65042) were analysed by using R (v3.0.2) by executing different packages, including LIMMA [71] and arrayQualityMetrics [72]. The data were read in and background corrected (method = "normexp" and offset $=1$ ) with functions from the R package LIMMA [71] from Bioconductor [73]. Quantile normalisation of the data was done between arrays. The duplicate probes mapping to the same gene were averaged ('avereps') and subsequently the lower percentile of probes were removed in a three-step procedure, 1) get the highest of the dark spots to get a base value, 2) multiply by 1.1 , and 3) the gene/probe must be expressed in each of the samples in the experimental condition [74].

\section{Statistical and functional genomics analysis}

To test the differences between the chicken lines (X and $\mathrm{Y}$ ) on each day separately, the following contrasts, Y.0-X0, Y.4-X.4, and Y.16-X.16, were generated within the LIMMA package [71]. DAVID [75] was used to perform Functional Annotation Clustering (FAC) for the three different contrasts, i.e. Y.0-X0, Y.4-X.4, and Y.16-X.16. The upand down-regulated genes were separately analysed.

To investigate the temporal patterns for each chicken line and to find similarities and dissimilarities, we have performed a maSigPro analysis with subsequent soft clustering (MFuzz package). Clustering of data is an important bioinformatic tool in transcriptomic time-analysis and can uncover structures buried in these large transcriptomic datasets. Soft clustering is less sensitive to noise, compared to hard clustering and soft clustering has two main advantages, by generating how well clusters represent genes and the overall relation between clusters $[76,77]$. MaSigPro is a R-package [78], especially generated to handle short time-series microarray gene expression data and to find genes, based on a regression based approach, which have significantly different expression profiles between treatments. The following settings were used, in p.vector the $\mathrm{Q}$ value was set at 0.01 and multiple testing correction was performed by using Benjamini Hochberg with minimum observation of 20 . For T.fit, which makes a stepwise regression fit for time series gene expression experiments, the 'backward' step method was used with 'alfa' at 0.05 . Filtering of genes based on r-square $(>0.8)$ was performed and 'vars' was set at 'each', generating as many significant genes extractions as variables in the general regression model.

\section{Availability of supporting data}

The transcriptomics data discussed in this study have been deposited in NCBI Gene Expression Omnibus (GEO; http://www.ncbi.nlm.nih.gov/geo/), and are accessible through GEO series accession no. GSE65042 (http://www.ncbi.nlm. nih.gov/geo/query/acc.cgi?acc=GSE65042).

The microbiota data discusses in this study have been deposited in figshare (http://figshare.com/), and are accessible through http://dx.doi.org/10.6084/m9.figshare. 1406903.

\section{Additional file}

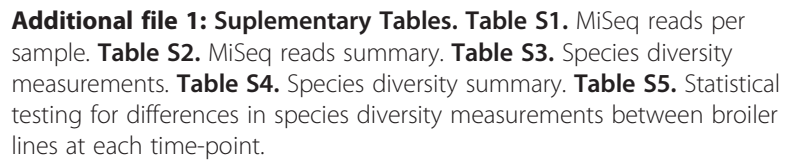

\section{Competing interests}

The authors declare that they have no competing interests.

\section{Authors' contributions}

DS carried out the statistical and bioinformatic analyses of both the gene expression and the microbiota, interpreted the data, and drafted the manuscript. SV carried out the laboratory work, i.e. DNA/RNA extraction and all work related to the microarrays (labelling, hybridisation, scanning). AB and FB carried out the bioinformatics and pre-processing regarding the microbiota (miSeq). GV, LK, MS, JR conceived of the study, and participated in its design and coordination and helped to critically revise the manuscript. All authors read and approved the final manuscript.

\section{Acknowledgements}

We would like to thank the animal care takers at the experimental farm and all the people who have helped in this project. Special thanks to the Breed4Food consortium for the funding of this project. 


\section{Author details}

${ }^{1}$ Wageningen Livestock Research, Wageningen, The Netherlands. ${ }^{2} \mathrm{Cobb}$ Europe BV, Boxmeer, The Netherlands. ${ }^{3}$ Central Veterinary Institute, Lelystad, The Netherlands.

Received: 28 January 2015 Accepted: 20 May 2015 Published online: 28 May 2015

\section{References}

1. Nauta AJ, Ben Amor K, Knol J, Garssen J, van der Beek EM. Relevance of preand postnatal nutrition to development and interplay between the microbiota and metabolic and immune systems. Am J Clin Nutr. 2013;98(2):586S-93.

2. Chung H, Pamp SJ, Hill JA, Surana NK, Edelman SM, Troy EB, et al. Gut immune maturation depends on colonization with a host-specific microbiota. Cell. 2012;149(7):1578-93.

3. El Aidy S, van Baarlen P, Derrien M, Lindenbergh-Kortleve DJ, Hooiveld G, Levenez $F$, et al. Temporal and spatial interplay of microbiota and intestinal mucosa drive establishment of immune homeostasis in conventionalized mice. Mucosal Immunol. 2012;5(5):567-79.

4. Cahenzli J, Koller Y, Wyss M, Geuking MB, McCoy KD. Intestinal microbial diversity during early-life colonization shapes long-term IgE levels. Cell Host Microbe. 2013;14(5):559-70.

5. Maynard $\mathrm{CL}$, Elson $\mathrm{CO}$, Hatton $\mathrm{RD}$, Weaver $\mathrm{CT}$. Reciprocal interactions of the intestinal microbiota and immune system. Nature. 2012;489(7415):231-41.

6. Musso G, Gambino R, Cassader M. Interactions between gut microbiota and host metabolism predisposing to obesity and diabetes. Annu Rev Med. 2011;62:361-80

7. Velagapudi VR, Hezaveh R, Reigstad CS, Gopalacharyulu P, Yetukuri L, Islam S, et al. The gut microbiota modulates host energy and lipid metabolism in mice. J Lipid Res. 2010;51(5):1101-12.

8. Bates JM, Mittge E, Kuhlman J, Baden KN, Cheesman SE, Guillemin K Distinct signals from the microbiota promote different aspects of zebrafish gut differentiation. Dev Biol. 2006;297(2):374-86.

9. Lutgendorff F, Akkermans LM, Soderholm JD. The role of microbiota and probiotics in stress-induced gastro-intestinal damage. Curr Mol Med. 2008;8(4):282-98.

10. Swann J, Wang Y, Abecia L, Costabile A, Tuohy K, Gibson G, et al. Gut microbiome modulates the toxicity of hydrazine: a metabonomic study. Mol Biosyst. 2009:5(4):351-5.

11. Buffie CG, Pamer EG. Microbiota-mediated colonization resistance against intestinal pathogens. Nature Rev. 2013:13(11):790-801.

12. Weng $M$, Walker WA: The role of gut microbiota in programming the immune phenotype. J Dev Orig Health Dis 2013, 4(3):203-14.

13. Apajalahti J, Kettunen A, Graham H. Characteristics of the gastrointestinal microbial communities, with special reference to the chicken. Worlds Poult Sci J. 2004;60(2):223-32.

14. Brisbin JT, Gong J, Sharif S. Interactions between commensal bacteria and the gut-associated immune system of the chicken. Anim Health Res Rev. 2008;9(1):101-10

15. Torok VA, Hughes RJ, Ophel-Keller K, Ali M, MacAlpine R. Influence of different litter materials on cecal microbiota colonization in broiler chickens. Poult Sci. 2009:88(12):2474-81.

16. Schokker D, Hoekman AJ, Smits MA, Rebel JM. Gene expression patterns associated with chicken jejunal development. Dev Comp Immunol. 2009;33(11):1156-64

17. Bar-Shira E, Friedman A. Development and adaptations of innate immunity in the gastrointestinal tract of the newly hatched chick. Dev Comp Immunol. 2006;30(10):930-41

18. Bar-Shira E, Sklan D, Friedman A. Establishment of immune competence in the avian GALT during the immediate post-hatch period. Dev Comp Immunol. 2003:27(2):147-57.

19. Sekelja M, Rud I, Knutsen SH, Denstadli V, Westereng B, Naes T, et al. Abrupt temporal fluctuations in the chicken fecal microbiota are explained by its gastrointestinal origin. Appl Environ Microbiol. 2012;78(8):2941-8.

20. Stanley D, Geier MS, Hughes RJ, Denman SE, Moore RJ. Highly variable microbiota development in the chicken gastrointestinal tract. PLoS One. 2013;8(12):e84290.

21. Campbell JH, Foster CM, Vishnivetskaya T, Campbell AG, Yang ZK, Wymore A, et al. Host genetic and environmental effects on mouse intestinal microbiota. ISME J. 2012:6(11):2033-44.
22. Spor A, Koren O, Ley R. Unravelling the effects of the environment and host genotype on the gut microbiome. Nat Rev Microbiol. 2011;9(4):279-90.

23. Benson AK, Kelly SA, Legge R, Ma F, Low SJ, Kim J, et al. Individuality in gut microbiota composition is a complex polygenic trait shaped by multiple environmental and host genetic factors. Proc Natl Acad Sci U S A. 2010:107(44):18933-8.

24. Peterson LW, Artis D. Intestinal epithelial cells: regulators of barrier function and immune homeostasis. Nature Rev. 2014;14(3):141-53.

25. Forder RE, Howarth GS, Tivey DR, Hughes RJ. Bacterial modulation of small intestinal goblet cells and mucin composition during early posthatch development of poultry. Poult Sci. 2007:86(11):2396-403.

26. Mulder IE, Schmidt B, Lewis M, Delday M, Stokes CR, Bailey M, et al. Restricting microbial exposure in early life negates the immune benefits associated with gut colonization in environments of high microbial diversity. PLoS One. 2011;6(12):e28279.

27. Schokker D, Zhang J, Zhang LL, Vastenhouw SA, Heilig HG, Smidt H, et al. Early-life environmental variation affects intestinal microbiota and immune development in new-born piglets. PLoS One. 2014;9(6):e100040

28. Kelly D, King T, Aminov R. Importance of microbial colonization of the gut in early life to the development of immunity. Mutat Res. 2007:622(1-2):58-69.

29. Swaggerty CL, Pevzner IY, Lowry VK, Farnell MB, Kogut MH. Functional comparison of heterophils isolated from commercial broiler chickens. Avian Pathol. 2003;32(1):95-102

30. Ferro PJ, Swaggerty CL, Kaiser P, Pevzner IY, Kogut MH. Heterophils isolated from chickens resistant to extra-intestinal Salmonella enteritidis infection express higher levels of pro-inflammatory cytokine mRNA following infection than heterophils from susceptible chickens. Epidemiol Infect. 2004;132(6):1029-37.

31. Swaggerty $\mathrm{CL}$, Ferro PJ, Pevzner IY, Kogut MH. Heterophils are associated with resistance to systemic Salmonella enteritidis infections in genetically distinct chicken lines. FEMS Immunol Med Microbiol. 2005:43(2):149-54.

32. Swaggerty $C L$, Lowry VK, Ferro PJ, Pevzner IY, Kogut MH. Disparity in susceptibility to vancomycin-resistant Enterococcus organ invasion in commercial broiler chickens that differ in innate immune responsiveness. Food Agr Immunol. 2005:16(1):1-15.

33. Li XY, Swaggerty CL, Kogut MH, Chiang Hl, Wang Y, Genovese K, et al. Systemic response to Campylobacter jejuni infection by profiling gene transcription in the spleens of two genetic lines of chickens. Immunogenetics. 2012;64(1):59-69.

34. Li X, Swaggerty CL, Kogut MH, Chiang H, Wang Y, Genovese KJ, et al. The paternal effect of Campylobacter jejuni colonization in ceca in broilers. Poult Sci. 2008:87(9):1742-7.

35. Swaggerty $C L$, Pevzner IY, Kaiser $P$, Kogut MH. Profiling pro-inflammatory cytokine and chemokine mRNA expression levels as a novel method for selection of increased innate immune responsiveness. Vet Immunol Immunopathol. 2008;126(1-2):35-42.

36. Pfefferle PI, Renz $\mathrm{H}$. The mucosal microbiome in shaping health and disease F1000prime reports. 2014:6:11.

37. Sommer F, Backhed F. The gut microbiota-masters of host development and physiology. Nat Rev Microbiol. 2013;11(4):227-38.

38. Dominguez-Bello MG, Costello EK, Contreras M, Magris M, Hidalgo G, Fierer N et al. Delivery mode shapes the acquisition and structure of the initial microbiota across multiple body habitats in newborns. Proc Natl Acad Sci U S A. 2010;107(26):11971-5.

39. Hildebrand F, Nguyen TL, Brinkman B, Yunta RG, Cauwe B, Vandenabeele $P$, et al. Inflammation-associated enterotypes, host genotype, cage and interindividual effects drive gut microbiota variation in common laboratory mice. Genome Biol. 2013;14(1):R4

40. Goodrich JK, Waters JL, Poole AC, Sutter JL, Koren O, Blekhman R, et al. Human genetics shape the gut microbiome. Cell. 2014;159(4):789-99.

41. Swaggerty $\mathrm{CL}$, Pevzner IY, Kogut MH. Selection for pro-inflammatory mediators yields chickens with increased resistance against Salmonella enterica serovar Enteritidis. Poult Sci. 2014:93(3):535-44.

42. Gilmore MS, Lebreton F, van Schaik W. Genomic transition of enterococci from gut commensals to leading causes of multidrug-resistant hospital infection in the antibiotic era. Curr Opin Microbiol. 2013;16(1):10-6.

43. Mundt JO. Occurrence of enterococci in animals in a wild environment Appl Microbiol. 1963;11:136-40

44. Hollenbeck BL, Rice LB. Intrinsic and acquired resistance mechanisms in enterococcus. Virulence. 2012;3(5):421-33. 
45. Schierack P, Walk N, Reiter K, Weyrauch KD, Wieler LH. Composition of intestinal Enterobacteriaceae populations of healthy domestic pigs. Microbiology. 2007;153(Pt 11):3830-7.

46. Pascual M, Hugas M, Badiola Jl, Monfort JM, Garriga M. Lactobacillus salivarius CTC2197 prevents Salmonella enteritidis colonization in chickens. Appl Environ Microbiol. 1999;65(11):4981-6.

47. Dalloul RA, Lillehoj HS, Shellem TA, Doerr JA. Enhanced mucosal immunity against Eimeria acervulina in broilers fed a Lactobacillus-based probiotic. Poult Sci. 2003;82(1):62-6.

48. La Ragione RM, Narbad A, Gasson MJ, Woodward MJ. In vivo characterization of Lactobacillus johnsonii Fl9785 for use as a defined competitive exclusion agent against bacterial pathogens in poultry. Lett Appl Microbiol. 2004;38(3):197-205.

49. Harty DWS, Oakey HJ, Patrikakis M, Hume EBH, Knox KW. Pathogenic potential of lactobacilli. Int J Food Microbiol. 1994;24(1-2):179-89.

50. Berggren A, Lazou Ahren I, Larsson N, Onning G. Randomised, double-blind and placebo-controlled study using new probiotic lactobacilli for strengthening the body immune defence against viral infections. Eur J Nutr. 2011;50(3):203-10.

51. Ebrahimi-Mameghani M, Sanaie S, Mahmoodpoor A, Hamishehkar H. Effect of a probiotic preparation (VSL\#3) in critically ill patients: a randomized, double-blind, placebo-controlled trial (pilot study). Pak J Med Sci. 2013;29(2):490-4.

52. Miele E, Pascarella F, Giannetti E, Quaglietta L, Baldassano RN, Staiano A. Effect of a probiotic preparation (VSL\#3) on induction and maintenance of remission in children with ulcerative colitis. Am J Gastroenterol. 2009;104(2):437-43.

53. Niedzielin K, Kordecki H, Birkenfeld B. A controlled, double-blind, randomized study on the efficacy of Lactobacillus plantarum $299 \mathrm{~V}$ in patients with irritable bowel syndrome. Eur J Gastroenterol Hepatol. 2001;13(10):1143-7.

54. Neal-McKinney JM, Lu X, Duong T, Larson CL, Call DR, Shah DH, et al. Production of organic acids by probiotic lactobacilli can be used to reduce pathogen load in poultry. PLoS One. 2012;7(9):e43928.

55. Mappley L, Tchorzewska MA, Nunez A, Woodward MJ, Bramley PM, La Ragione RM. Oral treatment of chickens with Lactobacillus reuteri LM1 reduces Brachyspira pilosicoli-induced pathology. J Med Microbiol. 2013;62(Pt 2):287-96.

56. Abudabos AM. Use of a competitive exclusion product (Aviguard (R)) to prevent clostridium perfringens colonization in broiler chicken under induced challenge. Pak J Zool. 2013;45(2):371-6.

57. Hall PA, Coates PJ, Ansari B, Hopwood D. Regulation of cell number in the mammalian gastrointestinal tract: the importance of apoptosis. J Cell Sci. 1994;107(Pt 12):3569-77.

58. Gunther C, Neumann H, Neurath MF, Becker C. Apoptosis, necrosis and necroptosis: cell death regulation in the intestinal epithelium. Gut. 2013;62(7):1062-71.

59. Yamauchi K, Kamisoyama H, Isshiki Y. Effects of fasting and refeeding on structures of the intestinal villi and epithelial cells in White Leghorn hens. $\mathrm{Br}$ Poultry Sci. 1996;37(5):909-21.

60. Burkholder KM, Thompson KL, Einstein ME, Applegate TJ, Patterson JA. Influence of stressors on normal intestinal microbiota, intestinal morphology, and susceptibility to Salmonella enteritidis colonization in broilers. Poult Sci. 2008:87(9):1734-41.

61. Teirlynck E, Bjerrum L, Eeckhaut V, Huygebaert G, Pasmans F, Haesebrouck F, et al. The cereal type in feed influences gut wall morphology and intestinal immune cell infiltration in broiler chickens. Brit J Nutr. 2009;102(10):1453-61.

62. Uni Z, Gal-Garber O, Geyra A, Sklan D, Yahav S. Changes in growth and function of chick small intestine epithelium due to early thermal conditioning. Poult Sci. 2001;80(4):438-45.

63. Arrieta MC, Bistritz L, Meddings JB. Alterations in intestinal permeability. Gut. 2006;55(10):1512-20.

64. Turner JR. Intestinal mucosal barrier function in health and disease. Nature Rev. 2009;9(11):799-809.

65. Keita AV, Soderholm JD. The intestinal barrier and its regulation by neuroimmune factors. Neurogastroenterol Motil. 2010;22(7):718-33.

66. Camilleri M, Madsen K, Spiller R, Greenwood-Van Meerveld B, Verne GN. Intestinal barrier function in health and gastrointestinal disease. Neurogastroenterol Motil. 2012;24(6):503-12.

67. Caporaso JG, Kuczynski J, Stombaugh J, Bittinger K, Bushman FD, Costello EK, et al. QIIME allows analysis of high-throughput community sequencing data. Nat Methods. 2010;7(5):335-6.
68. McDonald D, Price MN, Goodrich J, Nawrocki EP, DeSantis TZ, Probst A, et al. An improved Greengenes taxonomy with explicit ranks for ecological and evolutionary analyses of bacteria and archaea. ISME J. 2012;6(3):610-8.

69. DeSantis TZ, Hugenholtz P, Larsen N, Rojas M, Brodie EL, Keller K, et al. Greengenes, a chimera-checked 165 rRNA gene database and workbench compatible with ARB. Appl Environ Microbiol. 2006;72(7):5069-72.

70. Haas BJ, Gevers D, Earl AM, Feldgarden M, Ward DV, Giannoukos G, et al. Chimeric 165 rRNA sequence formation and detection in Sanger and 454-pyrosequenced PCR amplicons. Genome Res. 2011;21(3):494-504.

71. Gentleman R, Carey V, Huber W, Irizarry R, Dudoit S, Smyth GK. Limma: linear models for microarray data. In: Bioinformatics and Computational Biology Solutions Using R and Bioconductor. New York: Springer; 2005. p. 397-420.

72. Kauffmann A, Gentleman R, Huber W. ArrayQualityMetrics-a bioconductor package for quality assessment of microarray data. Bioinformatics. 2009;25(3):415-6.

73. Gentleman RC, Carey VJ, Bates DM, Bolstad B, Dettling M, Dudoit S, Ellis B, Gautier L, Ge Y, Gentry J et al: Bioconductor: open software development for computational biology and bioinformatics. Genome Biol 2004, 5(10).

74. Smyth GK: Linear models and empirical bayes methods for assessing differential expression in microarray experiments. Stat Appl Genet Mol Biol 2004, 3(1):1-25.

75. da Huang W, Sherman BT, Tan Q, Collins JR, Alvord WG, Roayaei J, et al. The DAVID gene functional classification tool: a novel biological module-centric algorithm to functionally analyze large gene lists. Genome Biol. 2007;8(9):R183.

76. Kumar L, M EF. Mfuzz: a software package for soft clustering of microarray data. Bioinformation. 2007;2(1):5-7.

77. Futschik ME, Carlisle B. Noise-robust soft clustering of gene expression timecourse data. J Bioinform Comput Biol. 2005;3(4):965-88.

78. Conesa A, Nueda MJ, Ferrer A, Talón M. maSigPro: a method to identify significantly differential expression profiles in time-course microarray experiments. Bioinformatics. 2006;22(9):1096-102.

\section{Submit your next manuscript to BioMed Central and take full advantage of:}

- Convenient online submission

- Thorough peer review

- No space constraints or color figure charges

- Immediate publication on acceptance

- Inclusion in PubMed, CAS, Scopus and Google Scholar

- Research which is freely available for redistribution 